\title{
Exercise and the platelet activator calcium chloride both influence the growth factor content of platelet-rich plasma (PRP): overlooked biochemical factors that could influence PRP treatment
}

\author{
Bruce Hamilton, ${ }^{1}$ Johannes L Tol, ${ }^{2}$ Wade Knez, ${ }^{3}$ Hakim Chalabi ${ }^{4}$
}

\begin{abstract}
${ }^{1}$ High Performance Sport New Zealand, Millenium Institute of Sport and Health, Auckland, New Zealand

2Department of Sports Medicine, Aspetar; Qatar Orthopaedic and Sports Medicine Hospital, Doha, Qatar ${ }^{3}$ Department of Research and Education, Aspetar; Qatar Orthopaedic and Sports Medicine Hospital, Doha, Qatar ${ }^{4} \mathrm{CMO}$ Office, Qatar Orthopaedic and Sports Medicine Hospital, Qatar
\end{abstract}

Correspondence to Dr Bruce Hamilton, Sports Medicine, High Performance Sport NZ, AUT/Millenium Institute of Sport and Health, Mairangi Bay, Auckland 0632 New Zealand; bruce. hamilton@hpsnz.org.nz, bruce.hamilton1510@gmail. com

Received 24 October 2012 Revised 18 March 2013 Accepted 31 March 2013 Published Online First 15 June 2013

\section{ABSTRACT}

Background There is strong evidence that exercise affects platelet haemostasis factors, but this potential effect on growth factor concentrations in platelet-rich plasma (PRP) has never been studied. In addition, there is a paucity of studies focusing on the effects of activating agents used in conjunction with PRP. The first aim of this study was to evaluate the effect of exercise on platelet and platelet-derived growth factors

(PDGF)-AB, hepatocyte growth factor (HGF), insulin-like growth factor-1 (IGF-1) and vascular endothelial growth factor (VEGF) concentrations in PRP. The second aim was to study the effect of the activating agent calcium chloride $\left(\mathrm{CaCl}_{2}\right)$ on growth factor concentration in relation to different exercise states.

Methods Controlled laboratory study. Ten healthy participants performed $1 \mathrm{~h}$ of submaximal exercise with blood being withdrawn immediately pre, post and $18 \mathrm{~h}$ following. PRP was prepared in each condition in both an activated $\mathrm{CaCl}_{2}$ and non-activated form.

Concentrations of PDGF-AB, HGF, IGF-1 and VEGF were evaluated using standard ELISA systems.

Results Exercise had no significant effect on platelet concentration, but significantly suppressed both VEGF and PDGF-AB concentrations. Exercise state had no significant effect on IGF-1 or HGF concentration. Activation with $\mathrm{CaCl}_{2}$ resulted in a significant increase in PDGF-AB and IGF-1 concentrations, unchanged VEGF and significantly reduced HGF concentrations.

Conclusions Exercise significantly impacts on PDGFs in PRP with significantly reduced concentrations of VEGF and PDFG-AB. Furthermore, the activation of PRP with $\mathrm{CaCl}_{2}$ results in a differentiated $\mathrm{GF}$ release from platelets. These relevant factors can potentially influence outcome in daily clinical practice and are recommended to be accounted for in future study design.

\section{INTRODUCTION}

A recent meta-analysis on the efficacy of the widely used autologous platelet-rich plasma (PRP) for 14 musculoskeletal indications showed conflicting evidence for its use, predominantly because of the shortcomings in standardisation of study protocols and confounding factors affecting the platelet application. ${ }^{1}$ The authors strongly recommended basic science studies focusing on the optimal preparation, dosage, effects of activating agents and timing of injecting autologous blood products.

Despite its apparent clinical popularity and a steadily increasing number of clinical studies, ${ }^{2-6}$ there is still only limited understanding of the role of the cellular and extracellular elements, optimal concentrations of platelets, leucocytes and released growth factor (GF) dose, timing and activation. ${ }^{7-9}$ Platelet-derived GF (PDGF) are stored in $\alpha$-granules found within platelets and are released in a selective manner upon activation. ${ }^{10}{ }^{11}$ Platelet activation is dependent on specific platelet membrane glycoproteins binding to ligands, kinase activation $^{12}$ and cytoplasmic calcium influx from both the dense tubular system and extracellular milieu, ${ }^{13} 14$ and may be initiated in vivo by a range of factors including thrombin, ${ }^{15}$ calcium, collagen ${ }^{9}{ }^{14}$ and shear stress. ${ }^{16}$ Each platelet contains about $80 \alpha$-granules, which, in addition to GF, contain adhesive proteins, chemokines, fibrinolytic proteins and pro-coagulant molecules. In vitro, calcium and thrombin are routinely utilised to induce GF release from PRP; in clinical practice, preactivation of PRP is widely used. ${ }^{17-19}$ However, evidence and consensus on the therapeutic requirement for preinjection activation is lacking. ${ }^{20-24}$

GF released from the $\alpha$-granules of platelets are assumed to provide the regenerative benefits of PRP. Haematological studies have shown that exercise may affect platelet function with an increased release of platelet-derived pro-coagulant microparticles following exercise. ${ }^{16} 25$ Subsequently, it is possible that exercise may also influence GF release from platelets, and thereby affect the clinical efficacy of the PRP.

The first aim of this study was to evaluate the effect of exercise on platelet and PDGFs PDGF-AB, hepatocyte growth factor (HGF), insulin-like growth factor-1 (IGF-1) and vascular endothelial growth factor (VEGF) concentrations in PRP. The second aim was to study the effect of the activating agent calcium chloride $\left(\mathrm{CaCl}_{2}\right)$ on GF concentration in relation to different exercise states.

\section{METHODOLOGY \\ Participants}

Eleven Caucasian male patients were recruited for the study after a thorough explanation of all the risks and benefits of participation in the study. The patients were subsequently excluded from analysis if they were suffering from any type of injury or were taking any medications that would impact on the platelet function. The study protocol was approved by our Institutional Medical Ethics Committee. All patients provided written informed consent. 


\section{Experimental overview}

Each participant was asked to report to the exercise laboratory having abstained from exercise for $36 \mathrm{~h}$ and following a $12 \mathrm{~h}$ fast (with the exception of water). Upon arrival at the controlled environmental laboratory $\left(\sim 21^{\circ} \mathrm{C}, 40-60 \% \mathrm{RH}, 760-770 \mathrm{~mm} \mathrm{Hg}\right)$, body mass and sports participation were recorded. Resting blood pressure was measured and venous blood $(10 \mathrm{ml})$ sampled from an antecubital vein. Participants then undertook a modified ${ }^{26}$ submaximal cycling test ${ }^{27}$ on an electronically braked cycle ergometer (Lode Excalibur Sport, Groningen, the Netherlands) to predict maximal aerobic capacity and peak power output (PPO). A single researcher supervised all cycling tests.

After 7 days, the initial laboratory procedure was repeated with the identical fasting and exercise abstention protocol. Prior to exercising, participants had $54 \mathrm{ml}$ of blood withdrawn, which was immediately prepared as described below. The participants were then required to exercise at $50 \%$ of their estimated PPO (based upon the previous test) for $1 \mathrm{~h}$. Immediately upon the cessation of exercise, a further $54 \mathrm{ml}$ of blood was sampled. The participants were advised to abstain from exercise; $18 \mathrm{~h}$ following the exercise bout, each subject had a further $54 \mathrm{ml}$ of blood withdrawn for analysis.

\section{Haematological analysis}

The blood was sampled from the antecubital vein with $10 \mathrm{ml}$ drawn into an EDTA-coated tube for immediate analysis of the whole blood platelet count. All platelet analysis was completed using the CELL-DYN 3700 SL analyser (Coulter Count; Abbot Diagnostics, Chicago, USA).

PRP was prepared using the manufacturer's instructions from the GPS III centrifuge separation system (Biomet Recover, GPS III Platelet Separation System). In a $60 \mathrm{ml}$ syringe, $54 \mathrm{ml}$ of whole blood was added to $6 \mathrm{ml}$ of ACD-A anticoagulant, and immediately centrifuged for $15 \mathrm{~min}$ at $3200 \mathrm{rpm}$. The extraction of PRP and platelet-poor plasma (PPP) was completed based on the method outlined in the commercially available separation system.

The volumes of PRP and PPP were recorded and each sample was separated into $500 \mu \mathrm{l}$ aliquots with a portion utilised for platelet concentration (PC) analysis as shown above. The remaining samples were either immediately stored at $-80^{\circ} \mathrm{C}$ or underwent activation with $\mathrm{CaCl}_{2}(25 \mathrm{mM})$ for $1 \mathrm{~h}$ at $37^{\circ} \mathrm{C}$. Following the activation, the samples were centrifuged at $4000 \mathrm{rpm}, 4^{\circ} \mathrm{C}$ for $10 \mathrm{~min}$ and the fibrin clot was separated from the supernatant. The supernatant was aliquoted and frozen at $-80^{\circ} \mathrm{C}$ until analysis. GF levels of PDGF-AB, HGF, IGF-1 and VEGF in the PRP, both the non-activated and following in vitro activation, were evaluated by commercially available ELISA (R\&D systems, Oxon, UK). All inter- and intra-assay coefficients of variation were $<10 \%$.

\section{Statistical analysis}

All statistical analyses were performed using SPSS (V.19.0). Data were screened for outliers and deviation from normality, and hence no transformations were applied. In addition, different GFs (HGF, IGF, PDGF-AB and VEGF) in PPP and PRP, respectively, were analysed using a linear mixed model, including fixed effect terms for activation status (activated or not activated) and time group ( $0 \mathrm{~h}$ (resting), immediately postexercise and $18 \mathrm{~h}$ postexercise) and random effect terms for participants and residuals. The effect size (ES) was calculated as partial $\eta^{2}$. ES of $0.01,0.06$ and 0.14 indicate small, medium and large association, respectively. Where a significant effect was found, post hoc pair wise comparisons were performed. Statistical significance was accepted at $\mathrm{p}<0.05$.

\section{RESULTS}

One participant was excluded from the study due to concurrently utilising a platelet-inhibiting medication (clopidogrel), and subsequently the data from 10 participants (mean age 34.3\pm 3.2 years; mean body mass index $26.1 \pm 4.28 \mathrm{~kg} / \mathrm{m}^{2}$ ) were analysed. All participants were physically fit and regularly engaged in moderate-to-intense physical exercise.

Mean PC in whole blood was within expected ranges (271.9$\left.\pm 54.0 \times 10^{3} \mu \mathrm{l}\right)$, increased significantly in PRP (1065.3$\left.\pm 4144.8 \times 10^{3} \mu \mathrm{l}\right)$ and decreased significantly in PPP (43.2 $\pm 16.3 \times 10^{3} \mu \mathrm{l}$ ). Mean white blood cell concentration (WBC) increased significantly from baseline whole blood levels (7.34 $\left.\pm 1.72 \times 10^{3} \mu \mathrm{l}\right)$ in PRP $\left(34.06 \pm 11.06 \times 10^{3} \mu \mathrm{l}\right)$ and significantly decreased in PPP $\left(0.04 \pm 0.04 \times 10^{3} \mu \mathrm{l}\right)$. In the PRP, PC was not associated with HGF, VEGF or IGF-1 concentrations. PC was positively associated with PDGF-AB $(\mathrm{p}<0.001)$; with every one unit increase in PC, a 25.2 unit increase is associated with PDGF.

\section{Effect of exercise state on PRP}

There was no significant change in PRP PC from resting to the postexercise conditions (figure 1). The analysis showed that there was an overall effect of exercise state on PDGF-AB (F $\quad(2,41.9)=4.0 ; \quad \mathrm{p}=0.025 ; \quad \mathrm{ES}=0.311)$ and $\mathrm{VEGF}$ ( $\mathrm{F}(2,42.3)=3.7 ; \mathrm{p}=0.034 ; \mathrm{ES}=0.456)$, with significant suppression of both VEGF (resting vs $1 \mathrm{~h} \mathrm{p}=0.024$; and vs $18 \mathrm{~h} \mathrm{p}=0.001$ ) and PDGF-AB (resting vs $1 \mathrm{~h} \mathrm{p}=0.021$; and vs $18 \mathrm{~h} \mathrm{p}=0.003$ ) concentration (figure 2). Exercise state had no significant effect on IGF-1 and HGF concentrations.

\section{Effect of activation state on PRP}

There was an overall effect of activation state on PDGF-AB $(\mathrm{F}(1,41.5)=63.6 ; \mathrm{p}<0.001, \mathrm{ES}=0.886), \mathrm{HGF}(\mathrm{F}(1,42.1)=69.9$; $\mathrm{p}<0.001, \mathrm{ES}=0.752)$ and $\operatorname{IGF}(\mathrm{F}(1,42.2)=11.3 ; \mathrm{p}=0.002$, $\mathrm{ES}=0.584$ ), but not VEGF. The activation resulted in a significant increase in PDGF-AB (resting $\mathrm{p}=0.012 ; 1 \mathrm{~h} \mathrm{p}<0.001 ; 18 \mathrm{~h}$ $\mathrm{p}<0.001)$ and IGF-1 (1 h p=0.022; $18 \mathrm{~h} \mathrm{p}=0.010)$ concentrations. HGF concentrations were significantly reduced after activation (resting $\mathrm{p}<0.001 ; 1 \mathrm{~h} \mathrm{p}<0.001 ; 18 \mathrm{~h} \mathrm{p}<0.001$ ). Postactivation, no significant differences were found for VEGF concentrations (figure 3).

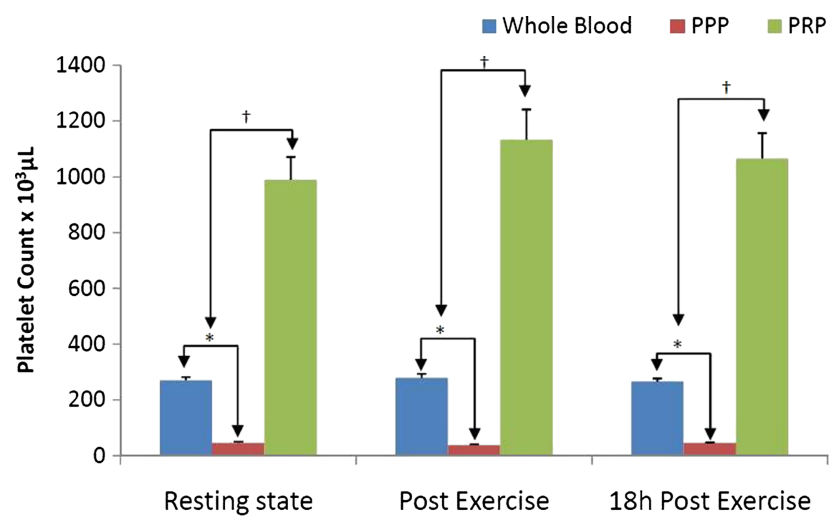

* Whole blood PC significantly higher than PPP $(p<0.001)$

† PRP PC significantly higher than Whole blood PC and PPP PC $(P<0.001)$

Figure 1 Platelet concentration (mean \pm SE) of platelet-rich plasma (PRP) at resting state, immediately postexercise and $18 \mathrm{~h}$ postexercise. 


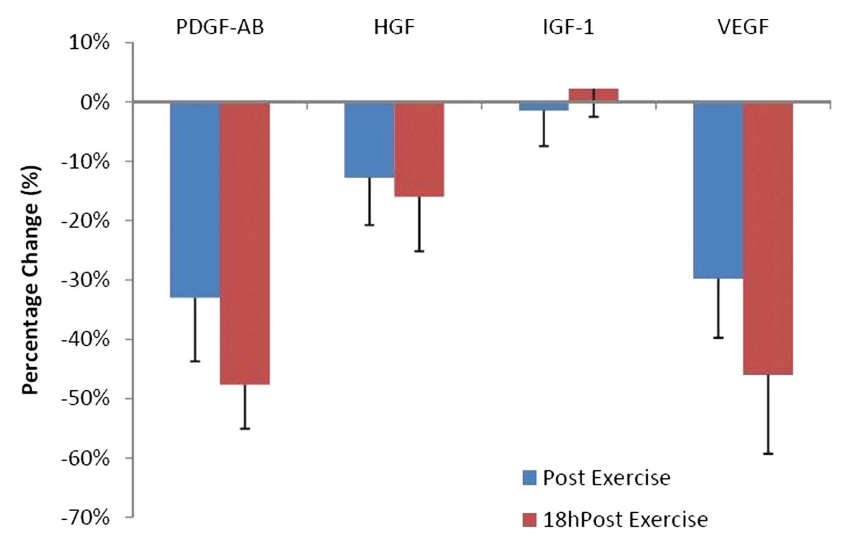

Figure 2 Effect of exercise state on platelet-rich plasma. Change in concentration of platelet-derived growth factors (PDGF)-AB, hepatocyte growth factor, insulin-like growth factor-1 and vascular endothelial growth factor (without preactivation) from resting state, immediately postexercise and $18 \mathrm{~h}$ postexercise (mean $\pm \mathrm{SE}$ ). Compared with the resting state, percentage increase or decrease was calculated from average means for the 10 participants.

There was a significant interaction effect between activation status and time on PDGF-AB (F $(1,41.5)=63.6 ; \mathrm{p}<0.001$; $\mathrm{ES}=0.312)$ and $\operatorname{VEGF}(\mathrm{F}(2,42.1)=4.1 ; \mathrm{p}=0.023 ; \mathrm{ES}=0.398)$.

\section{DISCUSSION}

This investigation suggests that exercise may influence GF concentration in PRP, and that the activation of PRP with $\mathrm{CaCl}_{2}$ results in a differentiated GF release. These are important observations since the assumption is that the GF present in PRP provides the regenerative stimulus to tissues. However, we still have only a limited understanding of the multitude of factors, which may impact on GF concentrations, and subsequently the outcomes of treatment with PRP. ${ }^{8}{ }^{28}$

The platelet counts, WBC and factor of increase of platelets observed in this study are comparable with earlier reports where the same separation system was used. ${ }^{28} 29$ Levels of $600000-$ 1000000 platelets/ $\mu \mathrm{l}$ are considered as being appropriate for PRP, but there is little clinical evidence as to what level is optimal. ${ }^{17}{ }^{30-33}$ In contrast to the previous studies, ${ }^{9}$ our finding that platelet count in PRP was not associated with three of four GF concentrations, casts doubt on the clinical relevance of absolute platelet count. Of note, the WBC was observed to increase in this preparation of PRP. The merits of either elevated or reduced WBC in PRP remain clinically indeterminant, and may ultimately depend on the clinical indication. ${ }^{28}$

While previous authors have reported a $25 \%$ increase in PC in whole blood immediately following strenuous exercise, no significant change was found in either whole blood or PRP PC following our exercise protocol. ${ }^{25}$ We did, however, observe a significant effect of exercise on GF concentrations within PRP. It is well recognised that platelet $\alpha$-granules are heterogeneous in their content, and that different stimuli may result in differential granule and factor release. ${ }^{10} 1134$ Furthermore, it has been previously illustrated that in response to the exercise-induced shear stress, there will be an increase in platelet-derived factors involved in haemostasis. ${ }^{16}{ }^{25}$ Our finding that exercise significantly reduced the circulating concentrations of PDGFs, VEGF and PDFG-AB, but had no effect on HGF concentrations, supports this differential release of contents from $\alpha$-granules. IGF-1, which is not a primary constituent of platelet $\alpha$-granules, was also unaffected by strenuous exercise. Based on this finding, it would appear that exercise may be a confounder to $\alpha$-granule release and exercise proximity should to be considered when preparing PRP and reporting clinical outcomes. It remains to be determined if a change in GF concentration of the magnitude demonstrated will be clinically relevant. Although quality evidence for the clinical use of PRP for chronic degenerative tendon indications is lacking, ${ }^{1}$ the potency of GFs in acute muscle injuries has not been studied yet and remains unknown. Factors such as the timing, dose and relative proportions of GF may be relevant; ${ }^{32} 3536$ anything that modifies the GF content of PRP may have a confounding effect on outcomes.

Both the requirement and rationale for preinfiltration activation of PRP in the clinical setting remain controversial. One school of thought suggests that activation prior to the application ensures optimal levels of GF release, ${ }^{37}$ while the contrasting argument suggests that the platelets will respond to the in vivo environment and release appropriate GF. While each of these arguments has a theoretical basis, there remains no clinical evidence to delineate an answer. Our data imply that the
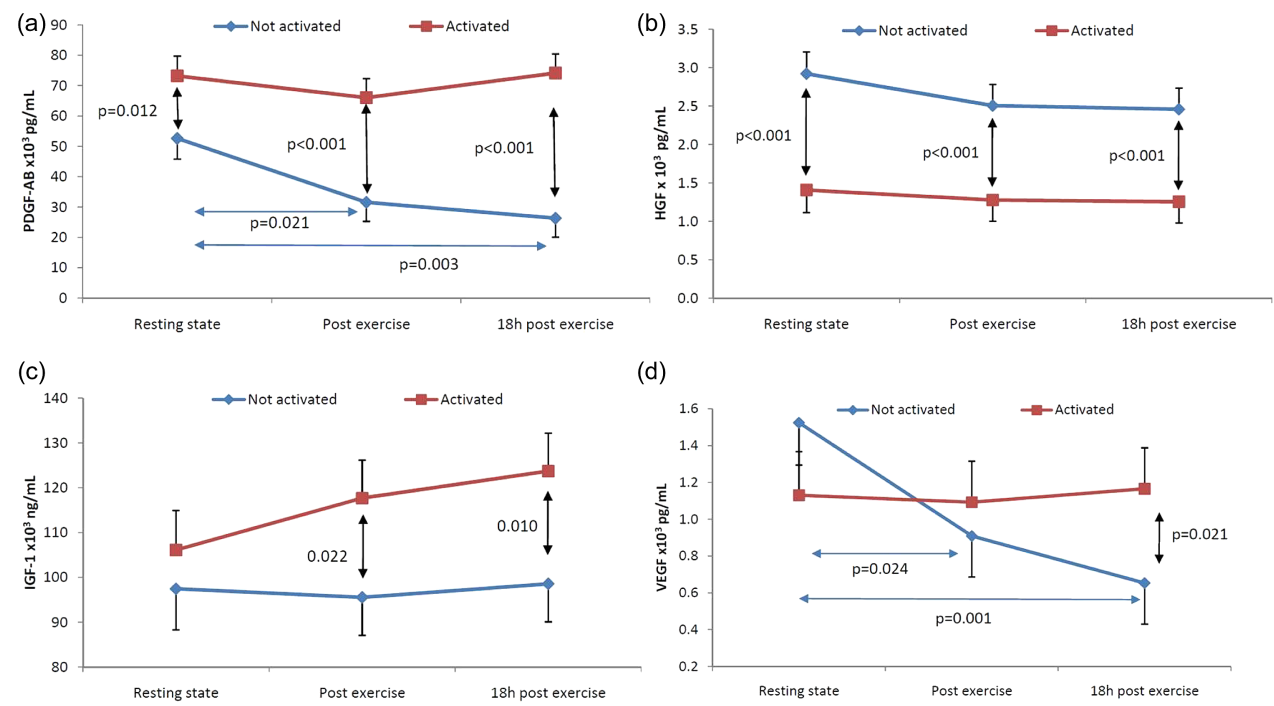

Figure 3 Effect of activation state. Platelet-derived growth factor (PDGF) concentrations PDGF-AB (A), hepatocyte growth factor (B), insulin-like growth factor-1 (C) and vascular endothelial growth factor (D) for the inactivated and activated state (mean $\pm \mathrm{SE}$ ). 
activation of PRP with $\mathrm{CaCl}_{2}$ results in a specific cellular response, with increased plasma concentrations of PDGF-AB and IGF-1, but paradoxically reduced concentrations of HGF and had no effect on VEGF concentrations. The cause of the paradoxical reduction in HGF concentration remains unknown. Although it cannot be excluded that HGF containing $\alpha$-granule release was inhibited by $\mathrm{CaCl}_{2}$, this would seem unlikely. We cannot rule out a technical error and further studies are required to test the reproducibility of the presented data. Similarly, IGF-1 is typically not considered a constituent of $\alpha$-granules. The mechanism by which activation with $\mathrm{CaCl}_{2}$ increased the IGF-1 concentration remains to be determined, but may be considered an extraplatelet effect. From a clinical perspective, the differential release of GF has no relationship to the clinical milieu in which the PRP will subsequently be infiltrated. A further study to delineate the results of preinfiltration activation with respect to the in vivo demands of the tissue is required.

An obvious limitation of this study is that only four GFs in 10 male participants were evaluated. Nonetheless, these GFs are among the most extensively studied ${ }^{9} 37$ and thought to play a prominent role in the regeneration process. With the chosen study design, it cannot be excluded that the fasting state, which was part of the resting state protocol, might have influenced GF concentrations. A control group without fasting is needed to clarify its potential confounding influence. A further confounder in this study may be that some GFs remained in the activated clot and were therefore not evaluated in the supernatant, thereby resulting in an underestimate of the actual GF release. The differential GF concentrations and a wide range of concentrations in response to both exercise and activation with $\mathrm{CaCl}_{2}$ suggest that further research in this area is required. As platelet function and effectiveness might be influenced by hormonal factors, validity in a female population remains unknown. We would recommend that in any future study design, exercise state be clearly defined.

\section{CONCLUSION}

This study illustrates that exercise may impact upon GF concentrations in PRP, with significantly reduced concentrations of VEGF and PDFG-AB. Furthermore, the activation of PRP with $\mathrm{CaCl}_{2}$ results in a differentiated GF release from platelets. These potentially confounding factors may influence clinical outcomes and should be accounted for both in future study design and when assessing clinical efficacy.

\section{What are the new findings?}

- This study illustrates that exercise may suppress both vascular endothelial growth factor and platelet-derived growth factor- $A B$ concentrations in PRP, thereby illustrating the importance of knowing the exercise state of subjects and patients when observing and reporting clinical outcomes.

- Furthermore, activation with $\mathrm{CaCl}_{2}$ resulted in a differential, non-uniform alteration in GF concentration. This latter finding is an important consideration for those researchers trying to establish the mechanisms behind any PRP efficacy, and highlights the complexity of this biological tool.

- This novel study adds important information for clinicians and researchers utilising PRP, and highlights the need for further detailed in vivo and in vitro research in a larger population.

\section{How might this study impact on clinical practice?}

In the future, the application of GF in the form of autologous blood products should be targeted to specific GFs at precise times in the healing process. This study highlights that exercise may affect the GF concentration, and therefore efficacy of PRP, and this may need to be considered in the future.

Acknowledgements The authors would like to acknowledge the invaluable support of Mohammed Farooq in the statistical analysis of this data.

Contributors All authors in this manuscript have made substantial contributions to the conception and design, acquisition of data or analysis and interpretation of data, drafting and revising it critically for important intellectual content; final approval of the version to be published.

Competing interests None.

Provenance and peer review Not commissioned; externally peer reviewed.

\section{REFERENCES}

1 Sheth U, Simunovic N, Klein G, et al. Efficacy of autologous platelet-rich plasma use for orthopaedic indications: a meta-analysis. J Bone Joint Surg 2012;94:298-307.

2 Gosens T, Peerbooms JC, van Laar W, et al. Ongoing positive effect of platelet-rich plasma versus corticosteroid injection in lateral epicondylitis: a double-blind reandomized controlled trial with 2-year follow-up. Am J Sports Med 2011;39:1200-8.

3 Thanasas C, Papadimitriou G, Charalambidis C, et al. Platelet-rich plasma versus autologous whole blood for the treatment of chronic lateral elbow epicondylitis: a randomized controlled clinical trial. Am J Sports Med 2011;39:2130-4.

4 de Vos $\mathrm{R}$, Weir $\mathrm{A}$, van Schie $\mathrm{H}$, et al. Platelet-rich plasma injection for chronic Achilles tendinopathy; a randomized controlled trial. JAMA 2010;303:144-9.

5 Castricini $R$, Longo U, De Benedetto $M$, et al. Platelet-rich plasma augmentation for arthroscopic rotator cuff repair: a randomized controlled trial. Am J Sports Med 2010;39:258-65.

6 Peerbooms JC, Sluimer J, Bruijn DJ, et al. Positive effect of an autologous platelet concentrate in lateral epicondylitis in a double-blind randomized controlled trial: platelet-rich plasma versus corticosteroid injection with a 1-year follow-up. Am J Sports Med 2010;38:255-62.

7 Creaney L. Platelet-rich plasma and the biological complexity of tissue regeneration. Br J Sports Med 2011;45:611.

8 Hamilton B, Best TM. Platelet-enriched plasma and muscle strain injuries: challenges imposed by the burden of proof. Clin J Sport Med 2011;21:31-6.

9 Harrison S, Vavken P, Kevy S, et al. Platelet activation by collagen provides sustained release of anabolic cytokines. Am J Sports Med 2011;39:729-34.

10 Italiano J, Battinelli E. Selective sorting of alpha-granule proteins. J Thromb Haemost 2009;7:173-6.

11 Italiano J, Richardson J, Patel-Hett S, et al. Angiogenesis is regulated by a novel mechanism: pro- and antiangiogenic proteins are organized into separate platelet alpha granules and differentially released. Blood 2008;111:1227-33.

12 Andrews R, Gardiner E, Asazuma N, et al. A novel viper venom metalloproteinase, alborhagin, is an agonist at the platelet collagen receptor GPVI. J Biol Chem 2001;276:28092-7.

13 Rendu F, Brohard-Bohn F. The platelet release reaction: granules' constituents, secretion and functions. Platelets 2001;12:261-73.

14 Roberts D, McNicol A, Bose R. Mechanism of collagen activation in human platelets. J Biol Chem 2004;279:19421-30.

15 Heijnen $H$, Schiel A, Fijnheer R, et al. Activated platelets release two types of membrane vesicles: microvesicles by surface shedding and exosomes derived from exocytosis of multivesicular bodies and alpha-granules. Blood 1999;94:3791-9.

16 Holme P, Orvim U, Hamers M, et al. Shear-induced platelet activation and platelet microparticle formation at blood flow conditions as in arteries with a severe stenosis. Arterioscler Thromb Vasc Biol 1997;17:646-53.

17 Anitua E, Sanchez A, Nurden A, et al. New insights into and novel applications for platelet-rich fibrin therapies. Trends Biotechnol 2006;24:227-34.

18 Anitua E, Sanchez E, Orive G, et al. Therapeutic applications of the preparation rich in growth factors (PRGF). Recent Adv Biomater Res 2008:141-53.

19 Anitua E, Sanchez M, Orive G, et al. The potential impact of the preparation rich in growth factors (PRGF) in different medical fields. Biomaterials 2007;28:4551-60.

20 Everts $\mathrm{P}$, Hoffmann J, Weibrich G, et al. Differences in platelet growth factor release and leucocyte kinetics during autologous platelet gel formation. Transf Med 2006;16:363-8. 
21 Everts $\mathrm{P}$, Mahoney $\mathrm{C}$, Hoffmann J, et al. Platelet-rich plasma preparation using three devices: Implications for platelet activation and platelet growth factor release. Growth Factors 2006;24:165-71.

22 Roussy Y, Duchesne M-P, Gagnon G. Activation of human platelet-rich plasmas: effect on growth factors release, cell division and in vivo bone formation. Clin Oral Implants Res 2007:18:639-48.

23 Zimmermann $R$, Jakubietz $R$, Jakubietz $M$, et al. Different preparation methods to obtain platelet components as a source of growth factors for local application. Transfusion 2001;41:1217-24.

24 Zimmermann R, Arnold D, Strasser E, et al. Sample preparation technique and white cell content influence the detectable levels of growth factors in platelet concentrates. Vox Sang 2003;85:283-9.

25 Chen Y-U, Chen J-K, Wang J-S. Strenuous exercise promotes shear-induced thrombin generation by increasing the shedding of procoagulant microparticles from platelets. Thromb Haemost 2010;104:293-301.

26 Legge B, Beanister E. The Astrand-Rhyming nomogram revisited. J Appl Physiol 1986;61:1203-9.

27 Astrand PO, Ryhming I. A Nomogram for calculation of aerobic capacity from pulse rate during submaximal work. J Appl Physiol 1954;7:218-21.

28 Castillo T, Pouliot M, Kim HL, et al. Comparison of growth factor and platelet concentration from commerscial platelet-rich plasma separation systems. Am J Sports Med 2011;39:266-71.
29 Hamilton B, Knez W, Eirale C, et al. Platelet enriched plasma for acute muscle injury. Acta Orthop Belg 2010;76:443-8.

30 Weibrich G, Hansen T, Kleis W, et al. Effect of platelet concentration in platelet-rich plasma on peri-implant bone regeneration. Bone 2004;34:665-71.

31 Marx R. Platelet-rich plasma (PRP): what is PRP and what is not PRP? Implant Dent 2001;10:225-8

32 Marx R. Platelet-rich plasma: evidence to support its use. J Oral Maxillofac Res 2004:62:489-96.

33 Lopez-Vidriero E, Goulding K, Simon D, et al. The use of platelt-rich plasma in arthroscopy and sports medicine: optimizing the healing environment. J Arthroscop Rel Surg 2010;26:269-78.

34 Liu Y, Kalen A, Risto O, et al. Fibroblast proliferation due to exposure to a platelet concentrate in vitro is $\mathrm{pH}$ dependent. Wound Repair and Regen 2002;10:336-40.

35 Chan B, Fu C, Qin L, et al. Supplementation-time dependence of growth factors in promoting tendon healing. ClinOrthop Relat Res 2006;448:240-7.

36 Hildebrand K, Woo S, Smith DJ, et al. The effects of platelet-derived growth factor-B on healing of the rabbit medial collateral ligament. An in vivo study. Am I Sports Med 1998;26:549-54.

37 Eppley B, Woodell J, Higgins J. Platelet quantification and growth factor analysis from platelet-rich plasma: implications for wound healing. Plast Reconstr Surg 2004;114:1502-8. 